\title{
On Characteristic Forms of Holomorphic Foliations
}

\author{
Yoshikatsu KAMOZAWA and Masahide KATO*
}

Sophia University

\section{Introduction.}

Let $M$ be a complex manifold of dimension $n(n \geq 2)$ and $\mathscr{F}$ a holomorphic foliation on $M$ of codimension $q(q \geq 1)$. We denote the normal bundle of $\mathscr{F}$ by $v(\mathscr{F})$, and its dual by $v(\mathscr{F})^{*}$. Then we can define the obstruction $P_{\mathscr{F}} \in H^{1}\left(M, v(\mathscr{F})^{*} \otimes \operatorname{End}(v(\mathscr{F}))\right)$ to the existence of holomorphic projective connection $\pi=\left\{p_{\alpha}\right\}$ of $v(\mathscr{F})$. As is well-known, there always exists a $C^{\infty}$ affine connection $a=\left\{a_{\alpha}\right\}$ of $v(\mathscr{F})$, by which we can define the Chern forms $\left\{c_{k}(a)\right\}_{k=1}^{q}$ of $v(\mathscr{F})$. Similarly there always exists a $C^{\infty}$ (normal reduced) projective connection $\pi=\left\{p_{\alpha}\right\}$ of $v(\mathscr{F})$, and this defines a kind of $C^{\infty}$ characteristic forms $\left\{P_{k}(\pi)\right\}_{k=1}^{q}$ of $v(\mathscr{F})$, which we call projective Weyl forms.

In this paper, we shall show that, for any $C^{\infty}$ normal reduced projective connection $\pi=\left\{p_{\alpha}\right\}$ of $v(\mathscr{F})$, the projective Weyl forms are $d$-closed, and that there exists a $C^{\infty}$ affine connection $a=\left\{a_{\alpha}\right\}$ of $v(\mathscr{F})$ which satisfies the following formulae;

$$
\begin{aligned}
& \sum_{k=0}^{q} c_{k}(a) t^{k}=\frac{(1+\alpha t)^{q+1}}{1+(\alpha-\beta) t} \sum_{k=0}^{q} P_{k}(\pi)\left(\frac{t}{1+\alpha t}\right)^{k}, \\
& \sum_{k=0}^{q} P_{k}(\pi) t^{k}=(1-\alpha t)^{q}(1-\beta t) \sum_{k=0}^{q} c_{k}(a)\left(\frac{t}{1-\alpha t}\right)^{k},
\end{aligned}
$$

where $c_{k}(a)$ is the $k$-th Chern form defined by the affine connection $a$, and both $\alpha$ and $\beta$ are $d$-closed 2-forms which represent the de Rham cohomology class $\left[\frac{1}{q+1} c_{1}(a)\right]$ (Theorem).

As a corollary to this theorem, in the cohomology class level, we get the formulae;

$$
\begin{aligned}
& \sum_{k=0}^{q}\left[c_{k}(a)\right] t^{k}=(1+[\alpha] t)^{q+1} \sum_{k=0}^{q}\left[P_{k}(\pi)\right]\left(\frac{t}{1+[\alpha] t}\right)^{k}, \\
& \sum_{k=0}^{q}\left[P_{k}(\pi)\right] t^{k}=(1-[\alpha] t)^{q+1} \sum_{k=0}^{q}\left[c_{k}(a)\right]\left(\frac{t}{1-[\alpha] t}\right)^{k} .
\end{aligned}
$$

\section{Received March 24, 1997}

Revised January 21, 1999

* Supported by the Grants-in-Aid for Scientific Research (C), the Ministry of Education, Science, Sports and Culture, 1998, Japan. 
These are generalizations of the results in [K1] to the case of holomorphic foliations on complex manifolds.

\section{Projective connections of a holomorphic foliation.}

Let $M$ be a complex manifold of dimension $n(n \geq 2)$. Let $\Gamma$ be the pseudogroup which is defined by the local biholomorphic maps of $\mathbf{C}^{q}$ and $\Gamma_{o}$ the pseudogroup defined by the local biholomorphic maps of $\mathbf{C}^{q}$ which fix the origin $o \in \mathbf{C}^{q}$. We denote by $j_{x}^{r}(f)$ the $r$-jet at $x$ of a mapping $f$ defined near the point $x$. In the following, we consider only 1 or 2 -jets, i.e., $r \leq 2$. Put

$$
P^{r}(q)=\left\{j_{x}^{r}(f): f \in \Gamma, x \in \mathbf{C}^{q}\right\}, \quad G^{r}(q)=\left\{j_{o}^{r}(f): f \in \Gamma_{o}\right\} .
$$

Then $G^{r}(q)$ is a complex Lie group with multiplication defined by the composition of jets,

$$
j_{o}^{r}(f) \cdot j_{o}^{r}(g)=j_{o}^{r}(f \circ g) .
$$

Definition 2.1. By a holomorphic foliation $\mathscr{F}$ of codimension $q(q \geq 1)$ on $M$, we shall mean a maximal system of 3-tuples $\left\{\left(U_{\alpha}, x_{\alpha}, \varphi_{\alpha \beta}\right)\right\}$ such that

1. $\left\{U_{\alpha}\right\}$ is an open covering of $M$,

2. $x_{\alpha}: U_{\alpha} \rightarrow \mathbf{C}^{q}$ is of maximal rank everywhere,

3. there is an element $\varphi_{\alpha \beta} \in \Gamma$ such that $x_{\alpha}=\varphi_{\alpha \beta}\left(x_{\beta}\right)$ on $U_{\alpha} \cap U_{\beta}$.

In what follows, $x_{\alpha}$ is called a local projection. The set

$$
P^{r}(\mathscr{F})=\left\{j_{x}^{r}\left(f_{\alpha}\right): f_{\alpha} \text { is a local projection with } f_{\alpha}(x)=o, x \in U_{\alpha}\right\}
$$

forms a principal fibre bundle over $M$ with the structure group $G^{r}(q)$, where $\pi: P^{r}(\mathscr{F}) \rightarrow$ $M, \pi\left(j_{x}^{r}\left(f_{\alpha}\right)\right)=x$, is the projection.

We shall describe our foliation in terms of local coordinates. Take an open covering $\mathscr{U}=\left\{U_{\alpha}\right\}$ of $M$ such that on each $U_{\alpha}$, there is a system of local coordinates $z_{\alpha}$ such as

$$
z_{\alpha}=\left(x_{\alpha}, y_{\alpha}\right)=\left(x_{\alpha}{ }^{1}, \cdots, x_{\alpha}{ }^{q}, y_{\alpha}{ }^{q+1}, \cdots, y_{\alpha}{ }^{n}\right)
$$

so that, for any leaf $L$, an arcwise connected component of $U_{\alpha} \cap L$ is given by $x_{\alpha}^{1}=c_{1}, \cdots, x_{\alpha}^{q}=c_{q}$ for some constants $c_{1}, \cdots, c_{q} \in \mathbf{C}$. Let $\varphi_{\alpha \beta}=\left(\varphi_{\alpha \beta}{ }^{j}, \varphi_{\alpha \beta}{ }^{\lambda}\right), 1 \leq j \leq q$, $q+1 \leq \lambda \leq n$, be a system of coordinate transformations on $U_{\alpha} \cap U_{\beta}$. More explicitly, we write

$$
\begin{aligned}
& x_{\alpha}^{j}=\varphi_{\alpha \beta}{ }^{j}\left(x_{\beta}{ }^{1}, \cdots, x_{\beta}{ }^{q}\right), \\
& y_{\alpha}{ }^{\lambda}=\varphi_{\alpha \beta}{ }^{\lambda}\left(x_{\beta}{ }^{1}, \cdots, x_{\beta}{ }^{q}, y_{\beta}{ }^{q+1}, \cdots, y_{\beta}{ }^{n}\right) .
\end{aligned}
$$

The transition function $T_{\alpha \beta}$ of the tangent bundle of $M$ is given by 


$$
T_{\alpha \beta_{k}}^{j}= \begin{cases}\frac{\partial \varphi_{\alpha \beta}{ }^{j}}{\partial x_{\beta}{ }^{k}} & (1 \leq k \leq q) \\ \frac{\partial \varphi_{\alpha \beta}{ }^{j}}{\partial y_{\beta}{ }^{k}} & (q+1 \leq k \leq n)\end{cases}
$$

We put

$$
\begin{gathered}
v_{\alpha \beta_{k}}{ }^{j}=\frac{\partial x_{\alpha}^{j}}{\partial x_{\beta}{ }^{k}}, \quad \tau_{\alpha \beta_{\mu}}^{\lambda}=\frac{\partial y_{\alpha}{ }^{\lambda}}{\partial y_{\beta}{ }^{\mu}}, \quad \tau_{\alpha \beta_{k}}{ }^{\lambda}=\frac{\partial y_{\alpha}{ }^{\lambda}}{\partial x_{\beta}{ }^{k}} \\
\left(\begin{array}{c}
1 \leq j, k \leq q \\
q+1 \leq \lambda, \mu \leq n
\end{array}\right) .
\end{gathered}
$$

Then

$$
T_{\alpha \beta}=\left(\begin{array}{cc}
v_{\alpha \beta} & 0 \\
\tau^{1}{ }_{\alpha \beta} & \tau_{\alpha \beta}
\end{array}\right) .
$$

The foliation $\mathscr{F}$ gives the exact sequence

$$
0 \rightarrow \Theta_{\mathscr{F}} \rightarrow \Theta_{M} \rightarrow v(\mathscr{F}) \rightarrow 0
$$

and its dual

$$
0 \rightarrow v(\mathscr{F})^{*} \rightarrow \Omega_{M}^{1} \rightarrow \Omega_{\mathscr{F}}^{1} \rightarrow 0,
$$

where we denote by $\Theta_{\mathscr{F}}$ the sheaf of germs of holomorphic vector fields tangential to the leaves of $\mathscr{F}, \Omega_{\mathscr{F}}^{1}$ the dual of $\Theta_{\mathscr{F}}, v(\mathscr{F})$ the sheaf of germs of holomorphic normal vectors to the leaves, and $v(\mathscr{F})^{*}$ the dual of $v(\mathscr{F})$. By the definition of $T_{\alpha \beta}$, it is clear that

$$
T_{\alpha \beta} T_{\beta \gamma}=T_{\alpha \gamma} .
$$

The principal bundle $P^{1}(\mathscr{F})$ is a $G^{1}(q)$-bundle associated with $v(\mathscr{F})$. We define also

$$
v_{\alpha \beta_{j k}}^{i}=\frac{\partial^{2} x_{\alpha}{ }^{i}}{\partial x_{\beta}{ }^{j} \partial x_{\beta}{ }^{k}} .
$$

An element $j_{x}^{2}(f) \in G^{2}(q)$ is described by the pair

$$
j_{x}^{2}(f)=\left(\left(f_{j}^{i}\right),\left(f_{j k}^{i}\right)\right) .
$$

Here $\left(f_{j}^{i}\right)$ is a $q \times q$ non-singular matrix, and $f_{j k}^{i}=f_{k j}^{i}$. The multiplication in $G^{2}(q)$ is given explicitly by

$$
j_{x}^{2}(f) \cdot j_{x}^{2}(g)=\left(\left(f_{j}^{i} g_{k}^{j}\right),\left(f_{k l}^{i} g_{r}^{k} g_{s}^{l}+f_{k}^{j} g_{r s}^{k}\right)\right) .
$$

The transition function $v_{\alpha \beta}$ of the normal bundle $v(\mathscr{F})$ defines an element

$$
j_{x_{\beta}}^{2}\left(v_{\alpha \beta}\right)=\left(\left(v_{\alpha \beta_{j}}^{i}\right),\left(v_{\alpha \beta_{j k}}^{i}\right)\right)
$$

for every $x \in U_{\alpha} \cap U_{\beta}$ with respect to the system of local coordinates $x_{\beta}$. The principal 
bundle $P^{2}(\mathscr{F})$ is the union

$$
\bigcup_{\alpha}\left(U_{\alpha} \times G^{2}(q)\right)
$$

where

$$
\left(x_{\alpha}, \xi_{\alpha}\right)=\left(x_{\alpha},\left(\xi_{\alpha_{j}}^{i}, \xi_{\alpha_{j k}}^{i}\right)\right) \in U_{\alpha} \times G^{2}(q)
$$

and

$$
\left(x_{\beta}, \xi_{\beta}\right)=\left(x_{\beta},\left(\xi_{\beta_{j}}^{i}, \xi_{\beta_{j k}}^{i}\right)\right) \in U_{\beta} \times G^{2}(q)
$$

are identified if and only if

$$
x_{\alpha}=x_{\beta}, \quad \xi_{\alpha}=j_{x_{\beta}}^{2}\left(v_{\alpha \beta}\right) \cdot \xi_{\beta} .
$$

The second equality is written more explicitly as

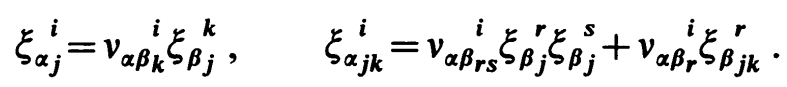

Motivated by Kobayashi-Nagano [KN, sections 5, 6], we define the subgroup $H^{2}(q)$ of $G^{2}(q)$ by

$$
H^{2}(q)=\left\{\left(\left(f_{j}^{i}\right),\left(f_{j k}^{i}\right)\right) \in G^{2}(q): f_{j k}^{i}=f_{j}^{i} \sigma_{k}+f_{k}^{i} \sigma_{j} \text { for some } \sigma_{j}\right\}
$$

For each transition function $v_{\alpha \beta}$ of the normal bundle $\mathscr{F}$, we define the following section $p_{\alpha \beta} \in \Gamma\left(U_{\alpha} \cap U_{\beta}, v(\mathscr{F})^{*} \otimes \operatorname{End}(v(\mathscr{F}))\right)$ by

$$
p_{\alpha \beta}=a_{\alpha \beta}-d x_{\beta}{ }^{i} \otimes \sigma_{\alpha \beta} \otimes \frac{\partial}{\partial x_{\beta}{ }^{i}}-\sigma_{\alpha \beta} \otimes d x_{\beta}{ }^{i} \otimes \frac{\partial}{\partial x_{\beta}{ }^{i}},
$$

where

$$
a_{\alpha \beta}=v_{\alpha \beta}^{-1}{ }_{m}^{i} v_{\alpha \beta_{j l}}^{m} d x_{\beta}^{j} \otimes d x_{\beta}^{l} \otimes \frac{\partial}{\partial x_{\beta}^{i}},
$$

$$
\sigma_{\alpha \beta}=\frac{1}{q+1} d \log \operatorname{det} v_{\alpha \beta} .
$$

Here $\partial / \partial x_{\alpha}{ }^{i} \in \Theta_{M}$ and its image by the natural projection $\Theta_{M} \rightarrow v(\mathscr{F})$ are indicated by the same symbol, and $i, j, k$ run over all $1 \leq i, j, k \leq q$. By an easy calculation, we have

Proposition 2.1. The set $\left\{a_{\alpha \beta}\right\}$ and $\left\{\sigma_{\alpha \beta}\right\}$ define cohomology classes such as

1. $\left\{a_{\alpha \beta}\right\} \in H^{1}\left(M, v(\mathscr{F})^{*} \otimes \operatorname{End}(v(\mathscr{F}))\right)$,

2. $\left\{\sigma_{\alpha \beta}\right\} \in H^{1}\left(M, v(\mathscr{F})^{*}\right)$.

The cohomology class defined by the set $\left\{a_{\alpha \beta}\right\}$, which we denote by $a_{\mathscr{F}}$, is well-defined by the foliation $\mathscr{F}$. The following is well-known.

Proposition 2.2. The structure group of the principal bundle $P^{2}(\mathscr{F})$ reduces to 
$G^{1}(q)$ if and only if $a_{\mathscr{F}}=0$.

If $a_{\mathscr{F}}=0$, then there is a 0 -cochain $a=\left\{a_{\alpha}\right\} \in C^{0}\left(\mathscr{U}, v(\mathscr{F})^{*} \otimes \operatorname{End}(v(\mathscr{F}))\right)$ such that $\delta\left\{a_{\alpha}\right\}=\left\{a_{\alpha \beta}\right\}$, which is called a holomorphic affine connection of $v(\mathscr{F})$. The cohomology class $a_{\mathscr{F}}=\left\{a_{\alpha \beta}\right\} \in H^{1}\left(M, v(\mathscr{F})^{*} \otimes \operatorname{End}(v(\mathscr{F}))\right)$ is the obstruction to the existence of holomorphic affine connection of $v(\mathscr{F})$. If $\left(\varphi_{\alpha \beta}^{j}\right)_{1 \leq j \leq q}$ is an affine transformation, then the obstruction $a_{\mathscr{F}}=\left\{a_{\alpha \beta}\right\}$ vanishes.

Similarly, by Proposition 2.1, we see that the set $\left\{p_{\alpha \beta}\right\}$ also defines an element of $H^{1}\left(M, v(\mathscr{F})^{*} \otimes \operatorname{End}(v(\mathscr{F}))\right)$. This cohomology class, which we denote by $P_{\mathscr{F}}$, is welldefined by the foliation $\mathscr{F}$. We can show the following.

PROPOSITION 2.3. The structure group of the principal bundle $P^{2}(\mathscr{F})$ reduces to $H^{2}(q)$ if and only if $P_{\mathscr{F}}=0$.

Proof. Suppose that the structure group of $P^{2}(\mathscr{F})$ reduces to $H^{2}(q)$. We can assume that there are some functions $\left\{\sigma_{\alpha \beta_{j}}\right\}$ defined on $U_{\alpha} \cap U_{\beta}$ such that

$$
v_{\alpha \beta_{j k}}^{i}=v_{\alpha \beta_{j}}^{i} \sigma_{\alpha \beta_{k}}+v_{\alpha \beta_{k}}^{i} \sigma_{\alpha \beta_{j}} \text {. }
$$

This equation determines $\sigma_{\alpha \beta_{j}}$, and we have the holomorphic functions

$$
\sigma_{\alpha \beta_{j}}=\frac{1}{q+1}\left(v_{\alpha \beta}^{-1}\right)_{i}^{l} v_{\alpha \beta_{l k}}^{i}
$$

Then

$$
\begin{aligned}
p_{\alpha \beta} & =v_{\alpha \beta}^{-{ }^{i}{ }_{m}}\left(v_{\alpha \beta_{j}}{ }^{m} \sigma_{\alpha \beta_{l}}+v_{\alpha \beta_{l}}{ }^{m} \sigma_{\alpha \beta_{j}}\right) d x_{\beta}{ }^{j} \otimes d x_{\beta}{ }^{l} \otimes \frac{\partial}{\partial x_{\beta}{ }^{i}}-d x_{\beta}{ }^{i} \otimes \sigma_{\alpha \beta} \otimes \frac{\partial}{\partial x_{\beta}{ }^{i}}-\sigma_{\alpha \beta} \otimes d x_{\beta}{ }^{i} \otimes \frac{\partial}{\partial x_{\beta}{ }^{i}} \\
& =d x_{\beta}{ }^{i} \otimes \sigma_{\alpha \beta} \otimes \frac{\partial}{\partial x_{\beta}{ }^{i}}+\sigma_{\alpha \beta} \otimes d x_{\beta}{ }^{i} \otimes \frac{\partial}{\partial x_{\beta}{ }^{i}}-d x_{\beta}{ }^{i} \otimes \sigma_{\alpha \beta} \otimes \frac{\partial}{\partial x_{\beta}{ }^{i}}-\sigma_{\alpha \beta} \otimes d x_{\beta}{ }^{i} \otimes \frac{\partial}{\partial x_{\beta}{ }^{i}}=0 .
\end{aligned}
$$

Conversely, suppose that $P_{\mathscr{F}}=0$. Then there exists a 0 -cochain $\pi=\left\{p_{\alpha}\right\}$,

$$
p_{\alpha}=p_{\alpha_{j k}}^{i} d x_{\alpha}^{j} \otimes d x_{\alpha}{ }^{k} \otimes \frac{\partial}{\partial x_{\alpha}{ }^{i}}
$$

in $C^{0}\left(\mathscr{U}, v(\mathscr{F})^{*} \otimes \operatorname{End}(v(\mathscr{F}))\right)$, such that

$$
p_{\alpha \beta}=p_{\beta}-p_{\alpha} .
$$

Then, by (3),

$$
v_{\alpha \beta_{j k}}^{i}=-p_{\alpha_{r s}}^{i} v_{\alpha \beta_{j}}^{r} v_{\alpha \beta_{k}}^{s}+v_{\alpha \beta_{r}}^{i} p_{\beta_{j k}}^{r}+v_{\alpha \beta_{j}}^{i} \sigma_{\alpha \beta_{k}}+\sigma_{\alpha \beta_{j}} v_{\alpha \beta_{k}}^{i}
$$

Hence

$$
\begin{aligned}
& \left(\left(\delta_{j}^{i}\right),\left(-p_{\alpha_{j k}}^{i}\right)\right)^{-1} \cdot\left(\left(v_{\alpha \beta_{j}}^{i}\right),\left(v_{\alpha \beta_{j k}}^{i}\right)\right) \cdot\left(\left(\delta_{j}^{i}\right),\left(-p_{\beta_{j k}}^{i}\right)\right) \\
& \quad=\left(\left(v_{\alpha \beta_{j}}^{i}\right),\left(v_{\alpha \beta_{m}}^{i} p_{\beta_{j k}}^{m}+p_{\alpha l m}^{i} v_{\alpha \beta_{j}}{ }^{l} v_{\alpha \beta_{k}}^{m}\right)\right)=\left(\left(v_{\alpha \beta_{j}}^{i}\right),\left(v_{\alpha \beta_{j}}^{i} \sigma_{\alpha \beta_{k}}+v_{\alpha \beta_{k}}^{i} \sigma_{\alpha \beta_{j}}\right)\right) .
\end{aligned}
$$


This shows that the structure group of $P^{2}(\mathscr{F})$ reduces to $H^{2}(q)$.

Definition 2.2. The 0-cochain $\pi=\left\{p_{\alpha}\right\} \in C^{0}\left(\mathscr{U}, v(\mathscr{F})^{*} \otimes \operatorname{End}(v(\mathscr{F}))\right)$ satisfying (5) is called a holomorphic projective connection of $v(\mathscr{F})$. The cohomology class $P_{\mathscr{F}}$ is called the obstruction to the existence of the holomorphic projective connection of $v(\mathscr{F})$.

We remark here that, if $\left(\varphi_{\alpha \beta}^{j}\right)_{1 \leq j \leq q}$ is a projective linear transformation, then $P_{\mathscr{F}}$ vanishes. We can also consider $C^{\infty}$ sections of the vector bundle $v(\mathscr{F})^{*} \otimes \operatorname{End}(v(\mathscr{F}))$. Let

$$
\xi_{\alpha}=\xi_{\alpha j k}^{i} d x_{\alpha}^{j} \otimes d x_{\alpha}^{k} \otimes \frac{\partial}{\partial x_{\alpha}^{i}}
$$

be a $C^{\infty}$ section of $v(\mathscr{F})^{*} \otimes \operatorname{End}(v(\mathscr{F}))$ defined on a local coordinate neighborhood $\left(U_{\alpha}, x_{\alpha}\right)$. In what follows, we write such section as a $q \times q$-matrix $\xi_{\alpha}=\left(\xi_{\alpha_{k}}^{i}\right)$, where the $(i, k)$-component is the $C^{\infty}(1,0)$-form defined by $\xi_{\alpha_{k}}^{i}=\xi_{\alpha_{j k}}^{i} d x_{\alpha}^{j}$. Using such matrix notation, the 1-cocycle $p_{\alpha \beta}$ of (3) is written as

$$
p_{\alpha \beta}=a_{\alpha \beta}-\rho_{\alpha \beta}-\sigma_{\alpha \beta} I,
$$

where $I$ is the $q \times q$ identity matrix, and

$$
\begin{aligned}
a_{\alpha \beta} & =v_{\alpha \beta}^{-1} d v_{\alpha \beta}, \\
\sigma_{\alpha \beta} & =\frac{1}{q+1} d \log \operatorname{det} v_{\alpha \beta}=\sum_{k=1}^{q} \sigma_{\alpha \beta_{k}} d x_{\beta}{ }^{k}, \\
\rho_{\alpha \beta} & =\left(\rho_{\alpha \beta_{k}}^{j}\right) \quad(1 \leq j, k \leq q), \\
\rho_{\alpha \beta_{k}}^{j} & =\sigma_{\alpha \beta_{k}} d x_{\beta}^{j} .
\end{aligned}
$$

By the matrix notation, holomorphic projective connection $\left\{p_{\alpha}\right\}$ of (5) is written as a set of $q \times q$-matrix valued holomorphic 1 -forms satisfying the equation

$$
p_{\alpha \beta}=p_{\beta}-v_{\alpha \beta}^{-1} p_{\alpha} v_{\alpha \beta} \text {. }
$$

In the following, we also consider $C^{\infty}$ projective connection. That is, a set $\left\{p_{\alpha}\right\}$ of $q \times q$-matrix valued $C^{\infty}(1,0)$-forms satisfying the same equation as (8).

DEFINITION 2.3. A $C^{\infty}$ projective connection $\left\{p_{\alpha}\right\}$ is said to be normal if $p_{\alpha_{j k}}^{i}=p_{\alpha_{k j}}^{i}$, and is said to be reduced if $p_{\alpha_{i k}}^{i}=0$.

Proposition 2.4. For any holomorphic foliation $\mathscr{F}$, there always exists a $C^{\infty}$ normal reduced projective connection of $v(\mathscr{F})$.

Proof. It is evident that there exists a $C^{\infty}$ projective connection $\pi=\left\{p_{\alpha}\right\}$. Given a $C^{\infty}$ projective connection $\pi=\left\{p_{\alpha}\right\}$, we can modify it to a normal and reduced one as follows. We put

$$
p_{\alpha \beta}=\left(p_{\alpha \beta j k}^{i} d x_{\beta}^{j}\right), \quad a_{\alpha \beta}=\left(a_{\alpha \beta j k}^{i} d x_{\beta}^{j}\right), \quad \rho_{\alpha \beta}=\left(\rho_{\alpha \beta j k}^{i} d x_{\beta}^{j}\right), \quad \sigma_{\alpha \beta}=\left(\delta_{j}^{i} \sigma_{\alpha \beta_{k}} d x_{\beta}^{j}\right) .
$$


By (6), we have

$$
p_{\alpha \beta_{j k}}^{i}=v_{\alpha \beta}^{-1^{i}}{ }_{l} \frac{\partial^{2} x_{\alpha}{ }^{l}}{\partial x_{\beta}{ }^{j} x_{\beta}{ }^{k}}-\delta_{j}^{i} \sigma_{\alpha \beta_{k}}-\delta_{k}^{i} \sigma_{\alpha \beta_{j}},
$$

where $l$ is summed over $1 \leq l \leq q$. Hence

$$
p_{\alpha \beta_{j k}}^{i}=p_{\alpha \beta_{k j}}^{i}
$$

Now we write $p_{\alpha}$ as

$$
p_{\alpha}=\left(p_{\alpha_{j k}}^{i} d x_{\alpha}^{j}\right)
$$

On the other hand, we define $q_{\alpha}$ by

$$
q_{\alpha}=\left(p_{\alpha_{k j}}^{i} d x_{\alpha}^{j}\right)
$$

The equation (8) is written as

$$
p_{\alpha \beta j k}^{i} d x_{\beta}^{j}=p_{\beta_{j k}}^{i} d x_{\beta}^{j}-v_{\alpha \beta}^{-1}{ }_{l}^{i} p_{\alpha r s}^{l} d x_{\alpha}^{r} v_{\alpha \beta_{k}}^{s} .
$$

Hence we have

$$
p_{\alpha \beta j k}^{i}=p_{\beta_{j k}}^{i}-v_{\alpha \beta}^{-1 i}{ }_{l}^{i} p_{\alpha r s}^{l} v_{\alpha \beta}{ }_{j}^{r} v_{\alpha \beta_{k}}^{s} \text {. }
$$

Therefore by (9), we have

$$
p_{\alpha \beta j k}^{i}=p_{\beta_{k j}}^{i}-v_{\alpha \beta}^{-1 i}{ }_{l}^{i} p_{\alpha r s}^{l} v_{\alpha \beta_{k}}^{r} v_{\alpha \beta_{j}}^{s}
$$

Multiplying (10) by $d x_{\beta}^{j}$, we have

$$
p_{\alpha \beta j k}^{i} d x_{\beta}^{j}=q_{\beta_{k}}^{i}-v_{\alpha \beta}^{-1}{ }_{l}^{i} q_{\alpha_{r}}^{l} v_{\alpha \beta_{k}}^{r} .
$$

Hence, we get

$$
p_{\alpha \beta}=q_{\beta}-v_{\alpha \beta}^{-1} q_{\alpha} v_{\alpha \beta} .
$$

Thus, by (8) and (11), if we replace $p_{\alpha}$ by $\frac{1}{2}\left(p_{\alpha}+q_{\alpha}\right)$, then $p_{\alpha}$ satisfies $p_{\alpha_{j k}}^{i}=p_{\alpha_{k j}}^{i}$. That is, the projective connection $\left\{p_{\alpha}\right\}$ can be always replaced with a normal one. In what follows, we assume that $\left\{p_{\alpha}\right\}$ is normal. By taking the traces of the equations (6) and (8), we have

$$
\left\{\begin{aligned}
\operatorname{tr} p_{\alpha \beta} & =\operatorname{tr} a_{\alpha \beta}-\operatorname{tr} \rho_{\alpha \beta}-\operatorname{tr}\left(\sigma_{\alpha \beta} I\right) \\
& =(q+1) \sigma_{\alpha \beta}-\sigma_{\alpha \beta}-q \sigma_{\alpha \beta}=0, \\
\operatorname{tr} p_{\alpha \beta} & =\operatorname{tr} p_{\beta}-\operatorname{tr} p_{\alpha} .
\end{aligned}\right.
$$

Hence we have

$$
\operatorname{tr} p_{\alpha}=\operatorname{tr} p_{\beta}
$$

Put $t_{\alpha}=\left(\operatorname{tr} p_{\alpha}\right) I$. Then

$$
t_{\alpha_{j}}^{i}=\delta_{j}^{i} \operatorname{tr} p_{\alpha}=\delta_{j}^{i} p_{\alpha_{k m}}^{m} d x_{\alpha}^{k}
$$


Define

$$
\left\{\begin{array} { r l } 
{ t _ { \alpha } ^ { \prime } } & { = ( t _ { \alpha _ { j } } ^ { \prime i } ) , } \\
{ t _ { \alpha _ { j } } ^ { \prime i } } & { = \delta _ { k } ^ { i } p _ { \alpha _ { j m } } ^ { m } d x _ { \alpha } ^ { k } , }
\end{array} \quad \left\{\begin{array}{r}
t_{\alpha}^{\prime \prime}=t_{\alpha}+t_{\alpha}^{\prime}, \\
t^{\prime \prime}\left(p_{\alpha}\right)=t_{\alpha}^{\prime \prime}
\end{array}\right.\right.
$$

Then by (12), $t^{\prime \prime}\left(p_{\alpha}\right)$ satisfies

$$
t^{\prime \prime}\left(p_{\alpha}\right)=v_{\beta \alpha}^{-1} t^{\prime \prime}\left(p_{\beta}\right) v_{\beta \alpha} .
$$

Note that $t^{\prime \prime}\left(p_{\alpha}\right)$ is normal. Since $\operatorname{tr}\left(t^{\prime \prime}\left(p_{\alpha}\right)\right)=(q+1) \operatorname{tr} p_{\alpha}$, replacing $\left\{p_{\alpha}\right\}$ with $\left\{p_{\alpha}-\frac{1}{q+1} t^{\prime \prime}\left(p_{\alpha}\right)\right\}$, we obtain a normal reduced projective connection of $v(\mathscr{F})$. Note that if $\left\{p_{\alpha}\right\}$ is holomorphic, then $\left\{p_{\alpha}-\frac{1}{q+1} t^{\prime \prime}\left(p_{\alpha}\right)\right\}$ is also holomorphic.

REMARK 1. It was pointed out by Fernanda Pambianco that the method described in [K1] and [K2] of replacing a given projective connection with a normal and reduced one contained a mistake. We can correct the mistake by the same argument as above.

REMARK 2. If the projective connection $\pi=\left\{p_{\alpha}\right\}$ is reduced, then in the case of $q=1, \pi=\left\{p_{\alpha}\right\}$ vanishes. Therefore, from now on, we shall consider the cases of $q \geq 2$.

\section{Weyl forms of a holomorphic foliation.}

Let $\mathscr{F}$ be a holomorphic foliation of codimension $q(q \geq 2)$ on $M$. From now on, to define Weyl forms, we calculate the Weyl curvature tensor of the normal bundle $v(\mathscr{F})$ of the holomorphic foliation $\mathscr{F}$ on $M$. Let $\pi=\left\{p_{\alpha}\right\}$ be a $C^{\infty}$ normal reduced projective connection of $v(\mathscr{F})$. From (6) and (8), we have

$$
\begin{aligned}
p_{\beta} & =a_{\alpha \beta}-\rho_{\alpha \beta}-\sigma_{\alpha \beta} I+v_{\alpha \beta}^{-1} p_{\alpha} v_{\alpha \beta} \\
& =v_{\alpha \beta}^{-1} d v_{\alpha \beta}-\rho_{\alpha \beta}-\sigma_{\alpha \beta} I+v_{\alpha \beta}^{-1} p_{\alpha} v_{\alpha \beta} .
\end{aligned}
$$

LEMMA 3.1.

(i) $d \sigma_{\alpha \beta}=0$,

(ii) $p_{\alpha} \wedge v_{\alpha \beta} \rho_{\alpha \beta}=0$,

(iii) $d v_{\alpha \beta} \wedge \rho_{\alpha \beta}=0$,

(iv) $\rho_{\alpha \beta} \wedge \rho_{\alpha \beta}=\rho_{\alpha \beta} \wedge \sigma_{\alpha \beta} I$,

(v) $\sigma_{\alpha \beta} I \wedge \sigma_{\alpha \beta} I=\rho_{\alpha \beta} \wedge \sigma_{\alpha \beta} I+\sigma_{\alpha \beta} I \wedge \rho_{\alpha \beta}$

$$
\begin{aligned}
& =v_{\alpha \beta}^{-1} d v_{\alpha \beta} \wedge \sigma_{\alpha \beta} I+\sigma_{\alpha \beta} I \wedge v_{\alpha \beta}^{-1} d v_{\alpha \beta} \\
& =v_{\alpha \beta}^{-1} p_{\alpha} v_{\alpha \beta} \wedge \sigma_{\alpha \beta} I+\sigma_{\alpha \beta} I \wedge v_{\alpha \beta}^{-1} p_{\alpha} v_{\alpha \beta}=0 .
\end{aligned}
$$

Proof. The equation (i) is obvious. The equations in (v) follow from the fact that $\rho_{\alpha \beta}, v_{\alpha \beta}^{-1} d v_{\alpha \beta}, v_{\alpha \beta}^{-1} p_{\alpha} v_{\alpha \beta}$ are 1-forms, and $\sigma_{\alpha \beta}$ is a scalar-valued 1-form. By the normality of $\left\{p_{\alpha}\right\}$, the $(i, m)$-component of $p_{\alpha} \wedge v_{\alpha \beta} \rho_{\alpha \beta}$ is 


$$
\begin{aligned}
p_{\alpha_{j}}^{i} \wedge v_{\alpha \beta_{l}}^{j} \rho_{\alpha \beta_{m}}^{l} & =p_{\alpha_{j k}}^{i} d x_{\alpha}{ }^{k} \wedge v_{\alpha \beta_{l}}^{j} \sigma_{\alpha \beta_{m}} d x_{\beta}{ }^{l} \\
& =\sigma_{\alpha \beta_{m}}\left(p_{\alpha_{j k}}^{i} d x_{\alpha}{ }^{k} \wedge d x_{\alpha}^{j}\right)=0 .
\end{aligned}
$$

Hence the equation (ii) follows. The $(i, m)$-component of $d v_{\alpha \beta} \wedge \rho_{\alpha \beta}$ is

$$
\begin{aligned}
d v_{\alpha \beta_{j}}{ }^{i} \wedge \rho_{\alpha \beta_{m}}^{j} & =\frac{\partial^{2} x_{\alpha}{ }^{i}}{\partial x_{\beta}{ }^{l} \partial x_{\beta}{ }^{j}} d x_{\beta}{ }^{l} \wedge \sigma_{\alpha \beta_{m}} d x_{\beta}{ }^{j} \\
& =\sigma_{\alpha \beta_{m}}\left(\frac{\partial^{2} x_{\alpha}{ }^{i}}{\partial x_{\beta}{ }^{l} \partial x_{\beta}{ }^{j}} d x_{\beta}{ }^{l} \wedge d x_{\beta}{ }^{j}\right)=0 .
\end{aligned}
$$

Hence we get (iii). The equation (iv) follows immediately from the definition.

From (2), we have

$$
\tau_{\alpha \beta}^{1} v_{\beta \gamma}+\tau_{\alpha \beta} \tau_{\beta \gamma}^{1}=\tau_{\alpha \gamma}^{1},
$$

where $\left\{\tau^{1}{ }_{\alpha \beta}\right\} \in H^{1}\left(M, \mathscr{H} o m\left(v(\mathscr{F}), \Theta_{\mathscr{F}}\right)\right)$. Let $\mathscr{H}_{o m^{\infty}}\left(v(\mathscr{F}), \Theta_{\mathscr{F}}\right)$ denote the sheaf of germs of $C^{\infty}$ homomorphisms $v(\mathscr{F}) \rightarrow \Theta_{\mathscr{F}}$. Since $H^{1}\left(M, \mathscr{H}_{o m^{\infty}}\left(v(\mathscr{F}), \Theta_{\mathscr{F}}\right)\right)=0$, there exists

$$
\left\{u_{\alpha}\right\} \in C^{0}\left(M, \mathscr{H}_{0 m^{\infty}}\left(v(\mathscr{F}), \Theta_{\mathscr{F}}\right)\right)
$$

such that

$$
\tau_{\alpha \beta}^{1}=\tau_{\alpha \beta} u_{\beta}-u_{\alpha} v_{\alpha \beta} .
$$

From Lemma 3.1 and (6) and (8), we have

$$
d p_{\beta}+p_{\beta} \wedge p_{\beta}=v_{\alpha \beta}^{-1}\left(d p_{\alpha}+p_{\alpha} \wedge p_{\alpha}\right) v_{\alpha \beta}-r_{\alpha \beta}
$$

with

$$
r_{\alpha \beta}=\rho_{\alpha \beta} \wedge v_{\alpha \beta}^{-1} d v_{\alpha \beta}+d \rho_{\alpha \beta}-\rho_{\alpha \beta} \wedge \sigma_{\alpha \beta} I+\rho_{\alpha \beta} \wedge v_{\alpha \beta}^{-1} p_{\alpha} v_{\alpha \beta}
$$

Here $r_{\alpha \beta}$ is a $q \times q$ matrix-valued $(2,0)$-form. Moreover we can write $r_{\alpha \beta}$ in the following form,

$$
r_{\alpha \beta}=\left(r_{\alpha \beta_{i}}^{h}\right)=\left(r_{\alpha \beta_{i j k}}^{h} d x_{\beta}^{j} \wedge d x_{\beta}^{k}\right)
$$

where

$$
r_{\alpha \beta_{i j k}}^{h}=\delta_{j}^{h} r_{\alpha \beta_{i k}}-\delta_{k}^{h} r_{\alpha \beta_{i j}}
$$

We rewrite (14) as

$$
\begin{aligned}
& d p_{\beta_{i k}}^{h} \wedge d x_{\beta}{ }^{k}+p_{\beta_{l j}}^{h} p_{\beta_{i k}}^{l} d x_{\beta}^{j} \wedge d x_{\beta}{ }^{k} \\
= & v_{\alpha \beta}^{-1{ }^{h}}\left(d p_{\alpha_{n t}}^{l} \wedge d x_{\alpha}{ }^{t}+p_{\alpha_{m s}}{ }^{l} p_{\alpha_{n t}}{ }^{m} d x_{\alpha}{ }^{s} \wedge d x_{\alpha}{ }^{t}\right) v_{\alpha \beta_{i}}{ }^{n}-r_{\alpha \beta_{i j k}}{ }^{h} d x_{\beta}{ }^{j} \wedge d x_{\beta}{ }^{k} .
\end{aligned}
$$

Comparing the terms containing $d y_{\beta}{ }^{\lambda}$, s in both hand sides of (16), we have 


$$
\frac{\partial p_{\beta_{i k}}^{h}}{\partial y_{\beta}^{\lambda}}=v_{\alpha \beta}^{-1}{ }_{l}^{h} \frac{\partial p_{\alpha_{n t}}^{l}}{\partial y_{\alpha}{ }^{\mu}} \tau_{\alpha \beta_{\lambda}}{ }^{\mu} v_{\alpha \beta_{k}}{ }^{t} v_{\alpha \beta_{i}}{ }^{n}
$$

while, comparing the terms containing no $d y_{\beta}{ }^{\lambda}$ 's in both hand sides of (16), we have

$$
\begin{aligned}
& \left(\frac{\partial p_{\beta_{i k}}^{h}}{\partial x_{\beta}^{j}}+p_{\beta_{l j}}^{h} p_{\beta_{i k}}^{l}\right) d x_{\beta}^{j} \wedge d x_{\beta}^{k} \\
& =\left\{v_{\alpha \beta}^{-1}{ }_{l}^{h}\left(\frac{\partial p_{\alpha_{n t}}^{l}}{\partial x_{\alpha}{ }^{s}} v_{\alpha \beta_{j}}{ }^{s} v_{\alpha \beta_{k}}^{t}+\frac{\partial p_{\alpha_{n t}}^{l}}{\partial y_{\alpha}{ }^{\mu}} \tau^{1}{ }_{\alpha \beta_{j}}^{\mu} v_{\alpha \beta_{k}}{ }^{t}+p_{\alpha_{m s}}^{l} p_{\alpha_{n t}}{ }^{m} v_{\alpha \beta_{j}}{ }^{s} v_{\alpha \beta_{k}}{ }^{t}\right) v_{\alpha \beta_{i}}{ }^{n}-r_{\alpha \beta_{i j k}}{ }^{h}\right\} \\
& \times d x_{\beta}^{j} \wedge d x_{\beta}^{k} \text {. }
\end{aligned}
$$

Now, recall the equation (13), i.e.,

$$
\tau_{\alpha \beta_{j}}^{1}=\tau_{\alpha \beta_{\lambda}}^{\mu} u_{\beta_{j}}^{\lambda}-u_{\alpha_{r}}^{\mu} v_{\alpha \beta_{j}}^{r}
$$

then (18) is written as

$$
\begin{aligned}
& \left(\frac{\partial p_{\beta_{i k}}^{h}}{\partial x_{\beta}{ }^{j}}+p_{\beta_{l j}}{ }^{h} p_{\beta_{i k}}^{l}-v_{\alpha \beta}^{-1}{ }_{l}{ }_{l}^{h} \frac{\partial p_{\alpha_{n t}}^{l}}{\partial y_{\alpha}{ }^{\mu}} \tau_{\alpha \beta}{ }_{\lambda}^{\mu} u_{\beta_{j}}{ }^{\lambda} v_{\alpha \beta_{k}}{ }^{t} v_{\alpha \beta_{i}}{ }^{n}\right) d x_{\beta}^{j} \wedge d x_{\beta}{ }^{k} \\
& =\left\{v_{\alpha \beta}^{-1}{ }_{l}{ }_{l}\left(\frac{\partial p_{\alpha_{n t}}{ }^{l}}{\partial x_{\alpha}{ }^{s}} v_{\alpha \beta_{j}}{ }^{s} v_{\alpha \beta_{k}}^{t}-\frac{\partial p_{\alpha_{n t}}^{l}}{\partial y_{\alpha}{ }^{\mu}} u_{\alpha_{r}}{ }^{\mu} v_{\alpha \beta_{j}}{ }^{r} v_{\alpha \beta_{k}}{ }^{t}+p_{\alpha_{m s}}^{l} p_{\alpha_{n t}}{ }^{m} v_{\alpha \beta_{j}} v_{\alpha \beta_{k}}{ }^{t}\right) v_{\alpha \beta_{i}}{ }^{n}-r_{\alpha \beta_{i j k}}{ }^{h}\right\} \\
& \times d x_{\beta}^{j} \wedge d x_{\beta}{ }^{k} \text {. }
\end{aligned}
$$

Using (17), the left hand side of (18) can be written as

$$
\left(\frac{\partial p_{\beta_{j k}}^{h}}{\partial x_{\beta}^{j}}+p_{\beta_{l j}{ }^{h}}^{h} p_{\beta_{i k}}^{l}-\frac{\partial p_{\beta_{i k}}^{h}}{\partial y_{\beta}{ }^{\lambda}} u_{\beta_{j}}^{\lambda}\right) d x_{\beta}^{j} \wedge d x_{\beta}{ }^{k}
$$

Put

$$
p_{\beta_{i j k}}^{\prime h}=\frac{\partial p_{\beta_{i k}}^{h}}{\partial x_{\beta}^{j}}+p_{\beta_{l j}}^{h} p_{\beta_{i k}}^{l}-\frac{\partial p_{\beta_{i k}}^{h}}{\partial y_{\beta}^{\lambda}} u_{\beta_{j}}^{\lambda}
$$

and

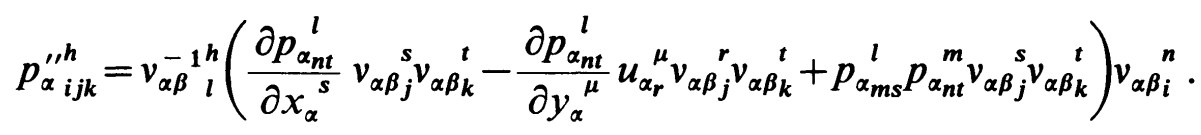

Then the equation (19) is written as

$$
p_{\beta_{i j k}}^{\prime h}-p_{\beta_{i k j}}^{\prime h}=p_{\alpha i j k}^{\prime \prime h}-p_{\alpha i k j}^{\prime \prime h}-\left(r_{\alpha \beta_{i j k}}^{h}-r_{\alpha \beta_{i k j}}^{h}\right) \text {. }
$$

Put

$$
X_{\beta_{i j k}}^{h}=p_{\beta_{i j k}}^{\prime h}-p_{\beta_{i k j}}^{\prime h} \text {. }
$$

Recall that the projective connection $\pi=\left\{p_{\alpha}\right\}$ is reduced. Contracting $X_{\beta_{i j k}}^{h}$ with respect to $h$ and $k$, we have 


$$
\begin{aligned}
X_{\beta_{i j}}: & =X_{\beta_{i j h}}^{h}=p_{\beta_{i j h}}^{\prime h}-p_{\beta_{i h j}}^{\prime h} \\
& =p_{\beta_{l j}}^{h} p_{\beta_{i h}}^{l}-\frac{\partial p_{\beta_{i j}}^{h}}{\partial x_{\beta}^{h}}+\frac{\partial p_{\beta_{i j}}^{h}}{\partial y_{\beta}{ }^{\lambda}} u_{\beta_{h}}^{\lambda} .
\end{aligned}
$$

Similarly, contracting the right hand side of (20) with respect to $h$ and $k$, we have

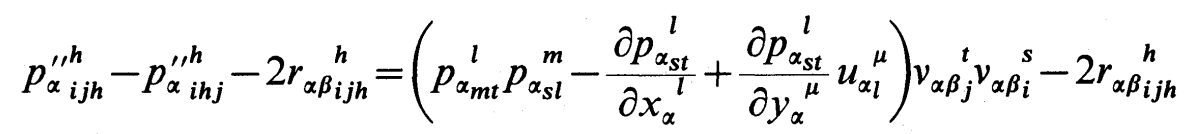

$$
\begin{aligned}
& =X_{\alpha_{s t}} v_{\alpha \beta_{j}}{ }^{t} v_{\alpha \beta_{i}}{ }^{s}-2 r_{\alpha \beta_{i j h}}{ }^{h} .
\end{aligned}
$$

Hence we get

$$
r_{\alpha \beta_{i j h}}^{h}=-\frac{1}{2}\left(X_{\beta_{i j}}-X_{\alpha_{s t}} v_{\alpha \beta_{j}}{ }^{t} v_{\alpha \beta_{i}}{ }^{s}\right) .
$$

From the equation (15), we have

$$
r_{\alpha \beta_{i j h}}^{h}=\delta_{j}^{h} r_{\alpha \beta_{i h}}-\delta_{h}^{h} r_{\alpha \beta_{i k}}=(1-q) r_{\alpha \beta_{i j}} .
$$

Hence

$$
r_{\alpha \beta_{i j}}=\frac{1}{2(q-1)}\left(X_{\beta_{i j}}-X_{\alpha_{s t}} v_{\alpha \beta_{j}}^{t} v_{\alpha \beta_{i}}^{s}\right)
$$

Therefore, from (18), we have

$$
\begin{aligned}
r_{\alpha \beta_{i j k}}{ }^{h} d x_{\beta}{ }^{j} \wedge d x_{\beta}{ }^{k} & =2 d x_{\beta}{ }^{h} \wedge r_{\alpha \beta_{i j}} d x_{\beta}{ }^{j} \\
& =\frac{1}{q-1} d x_{\beta}{ }^{h} \wedge\left(X_{\beta_{i j}}-X_{\alpha_{s t}} \nu_{\alpha \beta_{j}}{ }^{t} v_{\alpha \beta_{i}}{ }^{s}\right) d x_{\beta}{ }^{j} \\
& =\frac{1}{q-1} \delta_{l}^{h} X_{\beta_{i j}} d x_{\beta}{ }^{i} \wedge d x_{\beta}{ }^{j}-v_{\alpha \beta}{ }^{-1}{ }_{k}^{h}\left(\frac{1}{q-1} \delta_{l}^{k} X_{\alpha_{s t}} d x_{\alpha}{ }^{l} \wedge d x_{\alpha}{ }^{t}\right) v_{\alpha \beta_{i}}{ }^{s} .
\end{aligned}
$$

Thus if we put a tensor field $W=\left\{W_{\alpha}\right\}$ as

$$
\left\{\begin{aligned}
W_{\alpha} & =d p_{\alpha}+p_{\alpha} \wedge p_{\alpha}+\frac{1}{q-1} X_{\alpha}, \\
X_{\alpha} & =\delta_{j}^{h} X_{\alpha_{i k}} d x_{\alpha}^{j} \wedge d x_{\alpha}{ }^{k}, \\
X_{\alpha_{i k}} & =-\frac{\partial p_{\alpha_{i k}}^{h}}{\partial x_{\alpha}^{h}}+p_{\alpha l k}^{h} p_{\alpha_{i h}}^{l}+\frac{\partial p_{\alpha_{i k}}^{h}}{\partial y_{\alpha}{ }^{\lambda}} u_{\alpha_{h}}^{\lambda},
\end{aligned}\right.
$$

then we have

$$
W_{\beta}=v_{\alpha \beta}^{-1} W_{\alpha} v_{\alpha \beta} \text {. }
$$

Definition 3.1. The tensor field $W=\left\{W_{\alpha}\right\}$ defined as above is called the projective Weyl curvature tensor of the normal bundle $v(\mathscr{F})$ of the holomorphic foliation $\mathscr{F}$. 
Now we define a kind of characteristic $2 k$-forms, which we call Weyl forms. Let $t$ be an indeterminate and $A$ be an $n \times n$ matrix. Define polynomials $\varphi_{0}, \varphi_{1}, \cdots, \varphi_{n}$ by

$$
\operatorname{det}\left(I-\frac{t}{2 \pi i} A\right)=\sum_{k=0}^{n} \varphi_{k}(A) t^{k}
$$

By the equation (21), $P_{k}(\pi)=\varphi_{k}\left(W_{\alpha}\right)$ gives a $2 k$-form defined on the whole space $M$.

Definition 3.2. We call $P_{k}(\pi), 0 \leq k \leq q$, the $k$-th projective Weyl form of $v(\mathscr{F})$.

A priori, we do not know whether the $P_{k}(\pi)$ are $d$-closed or not. In the next section, we shall give a formula which relates the Chern forms of $v(\mathscr{F})$ with the Weyl forms of $v(\mathscr{F})$, which is our main theorem . By this formula we see that all the $P_{k}(\pi)$ are $d$-closed.

\section{Relations between the Chern forms and the Weyl forms.}

In this section, to show that the Weyl forms $\left\{P_{k}(\pi)\right\}$ are $d$-closed, we prove the following theorem, which is the main result of this paper. In what follows, the term "smooth" means $C^{\infty}$.

THEOREM. Let $M$ be a complex manifold of $\operatorname{dim}_{C} M=n(n \geq 2)$, and $\mathscr{F}$ a holomorphic foliation of codimension $q(q \geq 1)$ on $M$. Let $v(\mathscr{F})$ be a normal bundle of $\mathscr{F}$, and $\pi=\left\{p_{\alpha}\right\}$ any smooth normal reduced projective connection of $v(\mathscr{F})$. Then there exists a smooth affine connection $a=\left\{a_{\alpha}\right\}$ of $v(\mathscr{F})$, which satisfies the following equalities;

$$
\begin{gathered}
\sum_{k=0}^{q} c_{k}(a) t^{k}=\frac{(1+\alpha t)^{q+1}}{1+(\alpha-\beta) t} \sum_{k=0}^{q} P_{k}(\pi)\left(\frac{t}{1+\alpha t}\right)^{k}, \\
\sum_{i=0}^{q} P_{i}(\pi) t^{i}=(1-\alpha t)^{q}(1-\beta t) \sum_{k=0}^{q} c_{k}(a)\left(\frac{t}{1-\alpha t}\right)^{k},
\end{gathered}
$$

where $c_{k}(a)$ is the $k$-th Chern form associated with the affine connection $a=\left\{a_{\alpha}\right\}$ on $v(\mathscr{F})$, $P_{k}(\pi)$ is the $k$-th Weyl form associated with the projective connection $\pi=\left\{p_{\alpha}\right\}$ on $v(\mathscr{F})$, both $\alpha$ and $\beta$ are $d$-closed 2-forms which represent the de Rham cohomology class $\left[\frac{1}{q+1} c_{1}(a)\right]$. Here the equality $c_{1}(a)=\alpha+q \beta$ holds as forms.

REMARK 3. If the codimension $q$ equals 1 , then we cannot define the Weyl curvature tensor. But as we have seen before, the projective connection $\pi=\left\{p_{\alpha}\right\}$ vanishes for $q=1$. So the formulae above hold also in the case $q=1$.

As a corollary, we have

COROLlARY 4.1. In the cohomology level, we have the formulae as follows;

$$
\sum_{k=0}^{q}\left[c_{k}(a)\right] t^{k}=(1+[\alpha] t)^{q+1} \sum_{k=0}^{q}\left[P_{k}(\pi)\right]\left(\frac{t}{1+[\alpha] t}\right)^{k},
$$




$$
\sum_{i=0}^{q}\left[P_{i}(\pi)\right] t^{i}=(1-[\alpha] t)^{q+1} \sum_{k=0}^{q}\left[c_{k}(a)\right]\left(\frac{t}{1-[\alpha] t}\right)^{k},
$$

where $[\alpha]=\frac{1}{q+1}\left[c_{1}(a)\right]$.

To calculate the Chern forms of $v(\mathscr{F})$, we construct a smooth affine connection of $v(\mathscr{F})$, using the given $C^{\infty}$ normal reduced projective connection.

Now we denote by $K_{\mathscr{F}}$ the line bundle represented by the 1-cocycle $\left\{K_{\alpha \beta}\right\}$ such that

$$
\left\{\begin{array}{l}
K_{\mathscr{F}}=\left\{K_{\alpha \beta}\right\} \in H^{1}\left(M, \mathcal{O}^{*}\right), \\
K_{\alpha \beta}=\left(\operatorname{det} v_{\alpha \beta}\right)^{-1} .
\end{array}\right.
$$

Let $\left\{h_{\alpha}\right\}$ be a smooth metric of $\left\{K_{\alpha \beta}\right\}$, i.e.,

$$
h_{\beta}=\left|K_{\alpha \beta}\right|^{2} h_{\alpha} \quad \text { on } \quad U_{\alpha} \cap U_{\beta} \text {. }
$$

Then we have

$$
\begin{aligned}
\log h_{\beta} & =\log K_{\alpha \beta}+\log \bar{K}_{\alpha \beta}+\log h_{\alpha}, \\
\partial \log h_{\beta} & =\partial \log K_{\alpha \beta}+\partial \log h_{\alpha} .
\end{aligned}
$$

We define 1-cochains $\left\{\sigma_{\alpha}\right\}$ and $\left\{\rho_{\alpha}\right\}$ as follows:

DEFINITION 4.1.

$$
\begin{aligned}
& \sigma_{\alpha}=-\frac{1}{q+1} \partial \log h_{\alpha}=\sigma_{\alpha_{j}} d x_{\alpha}^{j}+\sigma_{\alpha_{\lambda}} d y_{\alpha}{ }^{\lambda}, \\
& \rho_{\alpha}=\left(\rho_{\alpha_{j}} d x_{\alpha}{ }^{k}\right), \\
& \rho_{\alpha_{j}}=\sigma_{\alpha_{j}}-\sigma_{\alpha_{\lambda}} u_{\alpha_{j}}^{\lambda} .
\end{aligned}
$$

Proposition 4.1.

(i) $\sigma_{\alpha \beta}=\sigma_{\beta}-\sigma_{\alpha}$,

(ii) $\rho_{\alpha \beta}=\rho_{\beta}-v_{\alpha \beta}^{-1} \rho_{\alpha} v_{\alpha \beta}$.

Proof. First we show (i). By Definition 4.1 and (4), we have immediately

$$
\begin{aligned}
\sigma_{\beta}-\sigma_{\alpha} & =-\frac{1}{q+1}\left(\partial \log h_{\beta}-\partial \log h_{\alpha}\right)=-\frac{1}{q+1} \partial \log K_{\alpha \beta} \\
& =-\frac{1}{q+1} \partial \log \left(\operatorname{det} v_{\alpha \beta}\right)^{-1}=\sigma_{\alpha \beta} .
\end{aligned}
$$

Next we show (ii). From (i) and Definition 4.1, we have by direct calculation, 


$$
\begin{aligned}
\sigma_{\alpha \beta} & =\sigma_{\beta}-\sigma_{\alpha}, \\
\sigma_{\alpha \beta_{j}} d x_{\beta}{ }^{j} & =\sigma_{\beta_{j}} d x_{\beta}{ }^{j}+\sigma_{\beta_{\lambda}} d y_{\beta}{ }^{\lambda}-\sigma_{\alpha_{i}} d x_{\alpha}{ }^{i}-\sigma_{\alpha_{\mu}} d y_{\alpha}{ }^{\mu} \\
& =\left(\sigma_{\beta_{j}}-\sigma_{\alpha_{i}} v_{\alpha \beta_{j}}{ }^{i}-\sigma_{\alpha_{\mu}} \tau^{1}{ }_{\alpha \beta_{j}}{ }^{\mu}\right) d x_{\beta}{ }^{j}+\left(\sigma_{\beta_{\lambda}}-\sigma_{\alpha_{\mu}} \tau_{\alpha \beta_{\lambda}}{ }^{\mu}\right) d y_{\beta}{ }^{\lambda} .
\end{aligned}
$$

Hence

$$
\left\{\begin{aligned}
\sigma_{\alpha \beta_{j}} & =\sigma_{\beta_{j}}-\sigma_{\alpha_{i}} \nu_{\alpha \beta_{j}}{ }^{i}-\sigma_{\alpha_{\mu}} \tau^{1}{ }_{\alpha \beta_{j}}{ }^{\mu} \\
\sigma_{\beta_{\lambda}} & =\sigma_{\alpha_{\mu}} \tau_{\alpha \beta_{\lambda}}{ }^{\mu}
\end{aligned}\right.
$$

Therefore, by (13),

$$
\begin{aligned}
\sigma_{\alpha \beta_{j}} & =\sigma_{\beta_{j}}-\sigma_{\alpha_{i}} v_{\alpha \beta_{j}}^{i}-\sigma_{\alpha_{\mu}}\left(\tau_{\alpha \beta_{\lambda}}^{\mu} u_{\beta_{j}}^{\lambda}-u_{\alpha_{l}}^{\mu} v_{\alpha \beta_{j}}{ }^{l}\right) \\
& =\left(\sigma_{\beta_{j}}-\sigma_{\alpha_{\mu}} \tau_{\alpha \beta_{\lambda}}{ }^{\mu} u_{\beta_{j}}^{\lambda}\right)-\left(\sigma_{\alpha_{i}} v_{\alpha \beta_{j}}{ }^{i}-\sigma_{\alpha_{\mu}} u_{\alpha l}^{\mu} v_{\alpha \beta_{j}}{ }^{l}\right) \\
& =\left(\sigma_{\beta_{j}}-\sigma_{\beta_{\lambda}} u_{\beta_{j}}^{\lambda}\right)-\left(\sigma_{\alpha_{i}}-\sigma_{\alpha_{\mu}} u_{\alpha_{i}}^{\mu}\right) v_{\alpha \beta_{j}}{ }^{i} .
\end{aligned}
$$

Since $d x_{\beta}{ }^{k}=v_{\alpha \beta}^{-1}{ }_{l}^{k} d x_{\alpha}{ }^{l}$, we have

$$
\begin{aligned}
\sigma_{\alpha \beta_{j}} d x_{\beta}{ }^{k} & =\left(\sigma_{\beta_{j}}-\sigma_{\beta \lambda} u_{\beta_{j}}^{\lambda}\right) d x_{\beta}{ }^{k}-\left(\sigma_{\alpha_{i}}-\sigma_{\alpha_{\mu}} u_{\alpha_{i}}^{\mu}\right) v_{\alpha \beta_{j}}{ }^{i} v_{\alpha \beta}^{-1}{ }_{l}{ }_{l} d x_{\alpha}{ }^{l} \\
& =\rho_{\beta_{j}} d x_{\beta}{ }^{k}-v_{\alpha \beta}{ }^{-1}{ }_{l}\left(\rho_{\alpha_{i}} d x_{\alpha}{ }^{l}\right) v_{\alpha \beta_{j}}{ }^{i} .
\end{aligned}
$$

Hence we get $\rho_{\alpha \beta}=\rho_{\beta}-v_{\alpha \beta}^{-1} \rho_{\alpha} v_{\alpha \beta}$. Thus (ii) is proved.

Now let $\pi=\left\{p_{\alpha}\right\}$ be a given smooth normal reduced projective connection of $v(\mathscr{F})$. Define a $q \times q$ matrix-valued smooth $(1,0)$-form $a_{\alpha}$ by

$$
a_{\alpha}=p_{\alpha}+\rho_{\alpha}+\sigma_{\alpha} I \text {. }
$$

Then we show that the 0 -cochain $a=\left\{a_{\alpha}\right\}$ is a smooth affine connection of $v(\mathscr{F})$. From (6), we can write $a_{\alpha \beta}$ as

$$
a_{\alpha \beta}=p_{\alpha \beta}+\rho_{\alpha \beta}+\sigma_{\alpha \beta} I \text {. }
$$

By (8) and Proposition 4.1, we get

$$
\begin{aligned}
a_{\alpha \beta} & =\left(p_{\beta}-v_{\alpha \beta}^{-1} p_{\alpha} v_{\alpha \beta}\right)+\left(\rho_{\beta}-v_{\alpha \beta}^{-1} \rho_{\alpha} v_{\alpha \beta}\right)+\left(\sigma_{\beta}-\sigma_{\alpha}\right) I \\
& =\left(p_{\beta}+\rho_{\beta}+\sigma_{\beta} I\right)-v_{\alpha \beta}^{-1}\left(p_{\alpha}+\rho_{\alpha}+\sigma_{\alpha} I\right) v_{\alpha \beta} \\
& =a_{\beta}-v_{\alpha \beta}^{-1} a_{\alpha} v_{\alpha \beta} .
\end{aligned}
$$

Thus we see that the 0 -cochain $a=\left\{a_{\alpha}\right\}$ is a smooth affine connection of $v(\mathscr{F})$. The curvature form $A=\left\{A_{\alpha}\right\}$,

$$
A_{\alpha}=d a_{\alpha}+a_{\alpha} \wedge a_{\alpha},
$$

satisfies the equation

$$
A_{\beta}=v_{\alpha \beta}^{-1} A_{\alpha} v_{\alpha \beta} .
$$


We try to write $A_{\alpha}$ in terms of $p_{\alpha}, \rho_{\alpha}$ and $\sigma_{\alpha} I$. By the definition of $\left\{\sigma_{\alpha}\right\}$, we have

$$
\begin{aligned}
d a_{\alpha} & =d p_{\alpha}+d \rho_{\alpha}+d \sigma_{\alpha} I=d p_{\alpha}+d \rho_{\alpha}+\bar{\partial} \sigma_{\alpha} I, \\
a_{\alpha} \wedge a_{\alpha} & =\left(p_{\alpha}+\rho_{\alpha}+\sigma_{\alpha} I\right) \wedge\left(p_{\alpha}+\rho_{\alpha}+\sigma_{\alpha} I\right) \\
& =p_{\alpha} \wedge p_{\alpha}+\rho_{\alpha} \wedge \rho_{\alpha}+p_{\alpha} \wedge \rho_{\alpha}+\rho_{\alpha} \wedge p_{a} .
\end{aligned}
$$

LEMMA 4.1.

$$
p_{\alpha} \wedge \rho_{\alpha}=d p_{\alpha} \wedge \rho_{\alpha}=p_{\alpha} \wedge d \rho_{\alpha}=0
$$

Proof. This follows from the normality of $\left\{p_{\alpha}\right\}$.

By Lemma 4.1 , we can write $A_{\alpha}$ as

$$
A_{\alpha}=d a_{\alpha}+a_{\alpha} \wedge a_{\alpha}=d p_{\alpha}+d \rho_{\alpha}+\bar{\partial} \sigma_{\alpha} I+p_{\alpha} \wedge p_{\alpha}+\rho_{\alpha} \wedge \rho_{\alpha}+\rho_{\alpha} \wedge p_{\alpha} .
$$

From now on we omit the subscript $\alpha$ for simplicity. Then the Chern forms of $v(\mathscr{F})$ are given by

$$
\left\{\begin{array}{l}
c_{k}(a)=\varphi_{k}(A) \\
\sum_{k=0}^{q} \varphi_{k}(A) t^{k}=\operatorname{det}\left(I_{q}-\frac{t}{2 \pi i} A\right)
\end{array}\right.
$$

Hence

$$
\begin{aligned}
\sum_{k=0}^{q} c_{k}(a) t^{k} & =\sum_{k=0}^{q} \varphi_{k}(A) t^{k}=\operatorname{det}\left(I_{q}-\frac{t}{2 \pi i} A\right) \\
& =\operatorname{det}\left(I_{q}-\frac{t}{2 \pi i}(d p+d \rho+\delta \sigma I+p \wedge p+\rho \wedge \rho+\rho \wedge p)\right)
\end{aligned}
$$

Put

$$
\lambda=1-\frac{t}{2 \pi i} d \sigma=1-\frac{t}{2 \pi i} \overline{\partial \sigma}
$$

Then by Lemma 4.1 , we get

$$
\begin{aligned}
I_{q}-\frac{t}{2 \pi i} A & =\lambda\left(I_{q}-\frac{t}{2 \pi i \lambda}(d p+d \rho+p \wedge p+\rho \wedge \rho+\rho \wedge p)\right) \\
& =\lambda\left(I_{q}-\frac{t}{2 \pi i \lambda}(d p+p \wedge p)\right)\left(I_{q}-\frac{t}{2 \pi i \lambda}(\rho \wedge p)\right)\left(I_{q}-\frac{t}{2 \pi i \lambda}(d \rho+\rho \wedge \rho)\right) .
\end{aligned}
$$

Hence the equation (22) becomes 


$$
\begin{aligned}
\sum_{k=0}^{q} c_{k}(a) t^{k}= & \operatorname{det}\left(I_{q}-\frac{t}{2 \pi i} A\right) \\
= & \lambda^{q} \operatorname{det}\left(I_{q}-\frac{t}{2 \pi i \lambda}(d p+p \wedge p)\right) \operatorname{det}\left(I_{q}-\frac{t}{2 \pi i \lambda}(\rho \wedge p)\right) \\
& \times \operatorname{det}\left(I_{q}-\frac{t}{2 \pi i \lambda}(d \rho+\rho \wedge \rho)\right)
\end{aligned}
$$

Proposition 4.2.

$$
\operatorname{det}\left(I_{q}-\frac{t}{2 \pi i \lambda}(d p+p \wedge p)\right)=\operatorname{det}\left(I_{q}-\frac{t}{2 \pi i \lambda} W\right)
$$

Proof. To prove the proposition, first we show the next lemma.

LEMMA 4.2.

$$
p \wedge X=d p \wedge X=0 \quad \text { where } \quad X=\left(\delta_{j}^{h} X_{i k} d x^{j} \wedge d x^{k}\right) .
$$

Proof. By the normality of $\left\{p_{\alpha}\right\}$, we have

$$
\begin{aligned}
(p \wedge X)_{j}^{i} & =p_{k}^{i} \wedge X_{j}^{k}=p_{k l}^{i} d x^{l} \wedge \delta_{m}^{k} X_{j s} d x^{m} \wedge d x^{s} \\
& =X_{j s} p_{k l}^{i} d x^{l} \wedge d x^{k} \wedge d x^{s}=0
\end{aligned}
$$

Similarly, we have $d p \wedge X=0$.

So we can write the right hand of (25) as

$$
\begin{aligned}
\operatorname{det}\left(I_{q}-\frac{t}{2 \pi i \lambda} W\right) & =\operatorname{det}\left(I_{q}-\frac{t}{2 \pi i \lambda}\left(d p+p \wedge p+\frac{1}{2(q-1)} X\right)\right) \\
& =\operatorname{det}\left\{\left(I_{q}-\frac{t}{2 \pi i \lambda}(d p+p \wedge p)\right)\left(I_{q}-\frac{t}{2 \pi i \lambda}\left(\frac{1}{2(q-1)} X\right)\right)\right\} \\
& =\operatorname{det}\left(I_{q}-\frac{t}{2 \pi i \lambda}(d p+p \wedge p)\right) \operatorname{det}\left(I_{q}-\frac{t}{2 \pi i \lambda}\left(\frac{1}{2(q-1)} X\right)\right) .
\end{aligned}
$$

LEMMA 4.3.

$$
\operatorname{det}\left(I_{q}-\frac{t}{2 \pi i \lambda} X\right)=1 \text {. }
$$

Proof. In general, we have the formula

$$
\operatorname{det}(I+t A)=\sum_{s=0}^{q}\left(\sum_{J_{s}}^{\prime} \operatorname{det}\left(A_{J_{s}}^{J_{s}}\right)\right) t^{s}
$$

where $J_{s}=\left\{\left(j_{1}, \cdots, j_{s}\right) \mid j_{1}<\cdots<j_{s}\right\}$. By this formula, we have 


$$
\operatorname{det}\left(I_{q}-\frac{t}{2 \pi i \lambda} X\right)=\sum_{s=0}^{q}\left(\sum_{J_{s}}^{\prime} \operatorname{det}\left(X_{J_{s}}^{J_{s}}\right)\right)\left(-\frac{t}{2 \pi i \lambda}\right)^{s}
$$

Then

$$
\begin{aligned}
\sum_{J_{s}}^{\prime} \operatorname{det}\left(X_{J_{s}}^{J_{s}}\right) & =\sum_{J_{s}}^{\prime} \sum_{\sigma \in S_{s}}(\operatorname{sgn} \sigma)\left(X_{\sigma(j)_{1} m_{1}} d x^{j_{1}} \wedge d x^{m_{1}} \wedge \cdots \wedge X_{\sigma(j)_{s} m_{s}} d x^{j_{s}} \wedge d x^{m_{s}}\right) \\
& =s ! \sum_{J_{s}}^{\prime} X_{j_{1} m_{1}} d x^{j_{1}} \wedge d x^{m_{1}} \wedge \cdots \wedge X_{j_{s} m_{s}} d x^{j_{s}} \wedge d x^{m_{s}} \\
& =\sum_{I_{s}} X_{i_{1} m_{1}} d x^{i_{1}} \wedge d x^{m_{1}} \wedge \cdots \wedge X_{i_{s} m_{s}} d x^{i_{s}} \wedge d x^{m_{s}} \\
& <\left(X_{i m} d x^{i} \wedge d x^{m}\right)^{s}
\end{aligned}
$$

where $I_{s}=\left\{\left(i_{1}, \cdots, i_{s}\right)\right\}$. Therefore the equation (26) is written as

$$
\begin{aligned}
\operatorname{det}\left(I_{q}-\frac{t}{2 \pi i \lambda} X\right) & =\sum_{s=0}^{q}\left(\sum_{J_{s}} \operatorname{det}\left(X_{J_{s}}^{J_{s}}\right)\right)\left(-\frac{t}{2 \pi i \lambda}\right)^{s} \\
& =\sum_{s=0}^{q}\left(X_{i m} d x^{i} \wedge d x^{m}\right)^{s}\left(-\frac{t}{2 \pi i \lambda}\right)^{s} .
\end{aligned}
$$

But, since $\left\{p_{\alpha}\right\}$ is normal, we see that

$$
X_{i m} d x^{i} \wedge d x^{m}=\left(-\frac{\partial p_{i m}^{h}}{\partial x^{h}}+p_{l m}^{h} p_{i h}^{l}+\frac{\partial p_{i m}^{h}}{\partial y^{\lambda}} u_{h}^{\lambda}\right) d x^{i} \wedge d x^{m}=0
$$

Hence we get

$$
\sum_{s=0}^{q}\left(X_{i m} d x^{i} \wedge d x^{m}\right)^{s}\left(-\frac{t}{2 \pi i \lambda}\right)^{s}=1
$$

Thus we have proved the lemma.

Proposition 4.2 follows from Lemmas 4.2 and 4.3.

Proposition 4.3.

$$
\operatorname{det}\left(I_{q}-\frac{t}{2 \pi i \lambda}(d \rho+\rho \wedge \rho)\right)=\left(1+\frac{t}{2 \pi i \lambda} \operatorname{tr} d \rho\right)^{-1} .
$$

Proof. Analogously to Lemma 4.3, the left hand side of (27) becomes

$$
\operatorname{det}\left(I_{q}-\frac{t}{2 \pi i \lambda}(d \rho+\rho \wedge \rho)\right)=\sum_{l=0}^{q}\left(d \rho_{j}^{j}+\rho_{k}^{j} \wedge \rho_{j}^{k}\right)^{\prime}\left(-\frac{t}{2 \pi i \lambda}\right)^{l} .
$$

Now we can easily see that 


$$
\begin{aligned}
d \rho_{j}^{j}+\rho_{k}^{j} \wedge \rho_{j}^{k} & =d \rho_{j}^{j}+\rho_{k} d x^{j} \wedge \rho_{j} d x^{k} \\
& =d \rho_{j}^{j}+\rho_{k} \rho_{j} d x^{j} \wedge d x^{k}=d \rho_{j}^{j}
\end{aligned}
$$

Therefore we have

$$
\operatorname{det}\left(I_{q}-\frac{t}{2 \pi i \lambda}(d \rho+\rho \wedge \rho)\right)=\sum_{s=0}^{q}(\operatorname{tr} d \rho)^{s}\left(-\frac{t}{2 \pi i \lambda}\right)^{s}=\left(1+\frac{t}{2 \pi i \lambda} \operatorname{tr} d \rho\right)^{-1} .
$$

Proposition 4.4. The 2-form $-\frac{q+1}{2 \pi i} \operatorname{tr} d \rho$ represents the Chern class $c_{1}\left(K_{\mathscr{F}}\right)$.

Proof. By taking the trace of the equation of Proposition 4.1 (ii), we have

$$
\sigma_{\alpha \beta}=\operatorname{tr} \rho_{\alpha \beta}=\operatorname{tr} \rho_{\beta}-\operatorname{tr} \rho_{\alpha} .
$$

Since

$$
-\frac{1}{2 \pi i} d \log K_{\alpha \beta}=-\frac{q+1}{2 \pi i} \sigma_{\alpha \beta},
$$

we have

$$
-\frac{1}{2 \pi i} d \log K_{\alpha \beta}=-\frac{q+1}{2 \pi i} \operatorname{tr} \rho_{\beta}+\frac{q+1}{2 \pi i} \operatorname{tr} \rho_{\alpha} .
$$

Hence, by de Rham theory, $\left\{-\frac{q+1}{2 \pi i} d \operatorname{tr} \rho_{\alpha}\right\}$ is a global 2-form on $M$ which represents the Chern class $c_{1}\left(K_{\mathscr{F}}\right)$.

Propsotion 4.5.

$$
\operatorname{det}\left(I_{q}-\frac{t}{2 \pi i \lambda}(\rho \wedge p)\right)=1
$$

Proof. Analogously to Lemma 4.3, we can prove the proposition as follows;

$$
\begin{aligned}
\operatorname{det}\left(I_{q}-\frac{t}{2 \pi i \lambda}(\rho \wedge p)\right) & =\sum_{s=0}^{q}\left(\rho_{k} d x^{j} \wedge p_{j}^{k}\right)^{s}\left(-\frac{t}{2 \pi i \lambda}\right)^{s} \\
& =\left(\rho_{k}^{j} \wedge p_{j}^{k}\right)^{0}\left(-\frac{t}{2 \pi i \lambda}\right)^{0}=1 .
\end{aligned}
$$

Combining Propositions 4.2, 4.3 and 4.5, we have from (24) that

$$
\begin{aligned}
\sum_{k=0}^{q} c_{k}(a) t^{k} & =\lambda^{q}\left(1+\frac{1}{2 \pi i} \operatorname{tr} d \rho\left(\frac{t}{\lambda}\right)\right)^{-1} \operatorname{det}\left(I_{q}-\frac{t}{2 \pi i \lambda} W\right) \\
& =\lambda^{q}\left(1+\frac{1}{2 \pi i} \operatorname{tr} d \rho\left(\frac{t}{\lambda}\right)\right)^{-1} \sum_{i=0}^{q} P_{i}(\pi)\left(\frac{t}{\lambda}\right)^{i} .
\end{aligned}
$$

Put 


$$
\alpha=-\frac{1}{2 \pi i} d \sigma \quad \text { and } \quad \beta=-\frac{1}{2 \pi i} \operatorname{tr} d \rho
$$

Then from (23), we have

$$
\begin{gathered}
\lambda=1+\alpha t \\
\sum_{k=0}^{q} c_{k}(a) t^{k}=\frac{(1+\alpha t)^{q+1}}{1+(\alpha-\beta) t} \sum_{i=0}^{q} P_{i}(\pi)\left(\frac{t}{1+\alpha t}\right)^{i} .
\end{gathered}
$$

If we put

$$
t^{\prime}=\frac{t}{\lambda}=\frac{t}{1+\alpha t}
$$

we have

$$
\sum_{i=0}^{q} P_{i}(\pi) t^{\prime i}=\left(1-\alpha t^{\prime}\right)^{q}\left(1-\beta t^{\prime}\right) \sum_{k=0}^{q} c_{k}(a)\left(\frac{t^{\prime}}{1-\alpha t^{\prime}}\right)^{k} .
$$

Note that, by definition,

$$
\begin{aligned}
c_{1}(a)= & \varphi_{1}(A)=-\frac{1}{2 \pi i} \operatorname{tr} A=-\frac{1}{2 \pi i} \operatorname{tr}(d a+a \wedge a) \\
& =-\frac{1}{2 \pi i} \operatorname{tr}(d a)=-\frac{1}{2 \pi i} \operatorname{tr}(d p+d \rho+d \sigma I)
\end{aligned}
$$

Since $\{p\}$ is reduced, $\operatorname{tr} d p=0$ holds. Hence we have

$$
-\frac{1}{2 \pi i} \operatorname{tr}(d p+d \rho+d \sigma I)=-\frac{1}{2 \pi i} \operatorname{tr}(d \rho+d \sigma I)=-\frac{1}{2 \pi i}(\operatorname{tr} d \rho+q d \sigma) .
$$

Thus we get $c_{1}(a)=\alpha+q \beta$. Thus we have proved our Theorem.

By Theorem, every $k$-th Weyl form $P_{k}(\pi)$ is expressed as a polynomial of the Chern forms $\left\{c_{j}(a)\right\}_{j=1}^{k}$. Since it is well-known that the $c_{j}(a)$ are $d$-closed, we have the following Corollary.

Corollary 4.2. The $k$-th Weyl form $P_{k}(\pi), 0 \leq k \leq q$, are $d$-closed.

Now we call the de Rham cohomology class $\left[P_{k}(\pi)\right] \in H^{2 k}(M, \mathrm{C})$ the $k$-th projective Weyl class. By the definition of $\alpha$ and $\beta$, we can easily see that $[\alpha]=[\beta]=\left[\frac{1}{q+1} c_{1}(a)\right]$.

COROLlary 4.3. In the cohomology level, we have

$$
\sum_{k=0}^{q}\left[c_{k}(a)\right] t^{k}=(1+[\alpha] t)^{q+1} \sum_{i=0}^{q}\left[P_{i}(\pi)\right]\left(\frac{t}{1+[\alpha] t}\right)^{i},
$$




$$
\sum_{i=0}^{q}\left[P_{i}(\pi)\right] t^{i}=(1-[\alpha] t)^{q+1} \sum_{k=0}^{q}\left[c_{k}(a)\right]\left(\frac{t}{1-[\alpha] t}\right)^{k},
$$

where $[\alpha]=\frac{1}{q+1}\left[c_{1}(a)\right]$.

Corollary 4.4. If a q-codimensional holomorphic foliation $\mathscr{F}$ on $M^{n}(n \geq 2)$ admits a holomorphic projective connection, then we have

$$
P_{k}(\pi)=0 \quad \text { for } \quad 2 k>q .
$$

Furthermore, if $M$ is compact and of Kähler, then also we have

$$
\boldsymbol{P}_{\boldsymbol{k}}(\mathscr{F})=\left[\boldsymbol{P}_{\boldsymbol{k}}(\pi)\right]=0 \quad \text { for } k \geq 1 .
$$

Proof. By the definition of the Weyl forms, if a foliation $\mathscr{F}$ on $M^{n}(n \geq 2)$ admits a holomorphic projective connection, then all $k$-th projective Weyl forms are holomorphic $2 k$-forms by (25). Therefore if $2 k>q$, then the $k$-th projective Weyl form vanishes. Since a $d$-closed holomorphic $q$-form represents a real de Rham cohomology class only if it represents a zero class, we see that the $q$-th projective Weyl class also vanishes. If, further, the manifold is compact and Kähler, then we can apply the Hodge theory. Since the projective Weyl forms are holomorphic, they are harmonic. On the other hand, by Corollary 4.2, the projective Weyl classes are real. Therefore they vanish by the Hodge theory.

\section{Examples.}

1. Let $T \mathbf{P}^{3}$ denote the tangent vector bundle of the complex projective 3-space $\mathbf{P}^{3}$ and $Z$ the associated projective bundle. On $Z$, we can consider two fibre bundle structures. One is the natural projection

$$
p: Z \rightarrow \mathbf{P}^{3}
$$

with fibers $\mathbf{P}^{2}$, and the other is the projection

$$
q: Z \rightarrow \operatorname{Gr}(4,2)
$$

to the Grassmannian manifold of all lines in $\mathbf{P}^{3}$ with fibers $\mathbf{P}^{1}$. The fibre of $q$ passing through a point $v \in Z$ corresponds to the line in $\mathbf{P}^{3}$ passing theough $p(v)$ with direction $v$. It is clear that, on $Z$, there is a holomorphic foliation $\mathscr{F}$ of codimension 3 with a holomorphic projective connection. Every leaf of $\mathscr{F}$ is a fibre of $p$, which is compact and biholomorphic to $\mathbf{P}^{2}$.

2. Obviously, every element of $P G L(4, C)$ induces a holomorphic automorphism of $Z$ and $\operatorname{Gr}(4,2)$. We fix two skew lines $l$ and $l^{\prime}$ in $\mathbf{P}^{3}$ (i.e., lines without intersection). Let $[l]$ be the point in $\operatorname{Gr}(4,2)$ defined by the line $l$ and $\left\{l^{\prime}\right\}$ the divisor in $\operatorname{Gr}(4,2)$ defined by 


$$
\left\{l^{\prime}\right\}=\left\{[L] \in \operatorname{Gr}(4,2) \text { : a line } L \subset \mathbf{P}^{3} \text { which intersects } l^{\prime}\right\} .
$$

Fix a system of homogeneous coordinates $\left[z_{0}: z_{1}: z_{2}: z_{3}\right]$ on $\mathbf{P}^{3}$ such that the two skew lines are given by

$$
\begin{aligned}
& l: z_{0}=z_{1}=0, \\
& l^{\prime}: \quad z_{2}=z_{3}=0 .
\end{aligned}
$$

Let $g \in P G L(4, \mathrm{C})$ be

$$
g=\left(\begin{array}{llll}
1 & 0 & 0 & 0 \\
0 & 1 & 0 & 0 \\
0 & 0 & 2 & 0 \\
0 & 0 & 0 & 2
\end{array}\right)
$$

Let

$$
W=Z-\left(q^{-1}\left([l] \cup\left\{l^{\prime}\right\}\right)\right) .
$$

Obviously, the infinite cyclic group $\langle g\rangle$ acts on $W$. The action is free and properly discontinuous. The quotient space $M=W /\langle g\rangle$ is a compact manifold of dimension 5 , which is a $\mathbf{P}^{1}$-bundle over $Y=\left(\operatorname{Gr}(4,2)-\left([l] \cup\left\{l^{\prime}\right\}\right)\right) /\langle g\rangle$. Here $Y$ is biholomorphic to a non-singular closed hypersurface of a 5-dimensional Hopf manifold. Let's see more closely the above argument using coordinates. Define the Plücker embedding of $\operatorname{Gr}(4,2)$ into $\mathbf{P}^{5}$ as follows. For a line in $\mathbf{P}^{3}$,

$$
\left\{\begin{array}{c}
a_{0} z_{0}+a_{1} z_{1}+a_{2} z_{2}+a_{3} z_{3}=0 \\
b_{0} z_{0}+b_{1} z_{1}+b_{2} z_{2}+b_{3} z_{3}=0
\end{array}\right.
$$

we define the Plücker coordinates by

$$
\left[\left|\begin{array}{ll}
a_{0} & a_{1} \\
b_{0} & b_{1}
\end{array}\right|:\left|\begin{array}{ll}
a_{0} & a_{2} \\
b_{0} & b_{2}
\end{array}\right|:\left|\begin{array}{ll}
a_{0} & a_{3} \\
b_{0} & b_{3}
\end{array}\right|:\left|\begin{array}{ll}
a_{1} & a_{2} \\
b_{1} & b_{2}
\end{array}\right|:\left|\begin{array}{ll}
a_{1} & a_{3} \\
b_{1} & b_{3}
\end{array}\right|:\left|\begin{array}{ll}
a_{2} & a_{3} \\
b_{2} & b_{3}
\end{array}\right|\right] .
$$

Then

$$
\operatorname{Gr}(4,2)=\left\{\left[\xi_{0}: \xi_{1}: \xi_{2}: \xi_{3}: \xi_{4}: \xi_{5}\right] \in \mathbf{P}^{5}: \xi_{0} \xi_{5}-\xi_{1} \xi_{4}+\xi_{2} \xi_{3}=0\right\},
$$

$[l]=[1: 0: 0: 0: 0: 0]$ in $\mathbf{P}^{5}$, and $\left\{l^{\prime}\right\}$ coincides with the hyperplane section $\operatorname{Gr}(4,2) \cap$ $\left\{\xi_{0}=0\right\}$. Therefore $\operatorname{Gr}(4,2)-\left\{l^{\prime}\right\}$ is a non-singular quadric in $\mathbf{C}^{5}=\mathbf{P}^{5}-\left\{\xi_{0}=0\right\}$. The automorphism (28) induces the following linear transformation $g$ on $\mathbf{C}^{5}$,

$$
g=\left(\begin{array}{lllll}
2 & 0 & 0 & 0 & 0 \\
0 & 2 & 0 & 0 & 0 \\
0 & 0 & 2 & 0 & 0 \\
0 & 0 & 0 & 2 & 0 \\
0 & 0 & 0 & 0 & 4
\end{array}\right)
$$


The set $G r(4,2)-\left\{l^{\prime}\right\}$ is $g$-invariant and the point [l] corresponds to the origin of $\mathbf{C}^{5}$. Therefore $Y=\left(\operatorname{Gr}(4,2)-\left([l] \cup\left\{l^{\prime}\right\}\right)\right) /\langle g\rangle$ is a submanifold of a 5-dimensional Hopf manifold. By our construction of $M$, we see that there is a holomorphic foliation $\mathscr{F}$ of codimension 3 with a holomorphic projective connection. Almost all leaves of $\mathscr{F}$ are non-compact, which are images of the fibers of $p^{-1}(x)-q^{-1}\left(\left\{l^{\prime}\right\}\right), x \in \mathbf{P}^{3}-l$. The leaves which are images of the fibers of $p^{-1}(x)-q^{-1}\left([l] \cup\left\{l^{\prime}\right\}\right), x \in l$, are compact and biholomorphic to Hopf surfaces.

\section{References}

[GH] P. Griffiths and J. Harris, Principles of Algebraic Geometry, Pure Appl. Math., Wiley (1978).

[KN] S. KobaYASHi and T. NAgANo, On projective connections, J. Math. Mech. 13 (1964), 215-235.

[K1] MA. Kato, On characteristic forms on complex manifold, J. Algebra 138 (1991), 424439.

[K2] MA. KATo, Logarithmic projective connections, Tokyo J. Math. 16 (1993), 99-119.

[T] I. TAMURA, Topology of Foliations, Iwanami (1976) (in Japanese).

Present Addresses:

YoshiKatSU KaMOZAWA

Nippon Telegraph and Telephone Corporation,

Konan, Minato-KU, TOKyo, 108-8019 JAPAN.

e-mail: kamozawa@2a.thj.tbn.ntt.co.jp

MaSAHIDE Kato

Department of Mathematics, Sophia University,

KIOICHO, ChIYODA-KU, TOKYO, 102-8554 JAPAN.

e-mail: kato@mm.sophia.ac.jp 